\title{
IBM PC Tachistoscope: Text stimuli
}

\author{
SIDNEY J. SEGALOWITZ \\ Brock University, St. Catharines, Ontario, Canada
}

\begin{abstract}
Due to cost, speed, and flexibility, the IBM PC and compatibles are good computers for laboratory use. Outlined here is a program that allows a computer from this class of machines to be used as a tachistoscope. The example given is for visual half-field presentation and can be easily modified for other presentation paradigms.
\end{abstract}

We have been using the IBM PC microcomputer and compatibles in a cognitive-perception laboratory for tachistoscopic presentation of text materials (see Diener \& Smee, 1984, for an Apple IIe tachistoscope program), and we have found that programming in BASIC is adequate for standard tachistoscopic presentation. The ease of using interpreted BASIC to try out the details of stimulus presentation makes this mode very useful indeed. A compiled version of the program is used for data collection. In the program presented here (Listing 1 ), only the routine to read the millisecond clock is programmed in assembly language. This language is needed if the experiment demands millisecond-accuracy reaction times and if the Tecmar clock ${ }^{1}$ is used. An alternative loop counter is also provided with slightly better than millisecond accuracy. The disadvantages of not using a real-time clock are described.

\section{THE PROGRAM STRUCTURE}

The program consists of a main program section and six GOSUB subroutines. Comments are included to help devise variations to suit specific experimental requirements. The example given in Listing 1 is for a visual halffield (VHF) study, where single words are presented on each trial. Minor reprogramming is needed to allow for double stimuli or other changes. The large-print format (40-character screen width) allows for a maximum of eight pages of text (i.e., eight viewing fields). In the program, the first page (SCREEN 0) is used for the fixation point, SCREEN 1 for the stimulus, SCREEN 2 for a postexposure blank field (onto which a mask can be placed), and SCREEN 3 for the end-of-experiment message. These can, of course, be varied.

\section{Stimulus Input}

For the configuration in Listing 1, the stimulus input file (STIMFIL $\$$ requested in line 100) should consist of stimulus word items separated by some delimeter, such as a comma, space, or carriage return. In general, however, a single stimulus can be up to a screenful of

This research was supported by a grant from the Natural Science and Engineering Research Council of Canada. Please address correspondence to the author at: Department of Psychology, Brock University, St. Catharines, Ontario L2S 3A1, Canada. text (40 characters $\times 25$ lines). It is up to the user to place the stimulus appropriately on the display (lines 360-420 in Listing 1). The program in Listing 1 places a word stimulus to the right or left of the fixation point, and accordingly each stimulus line in the input file contains a " 1 " or " 2 " (to indicate whether the LVF or RVF is to be used on that trial) and a word stimulus (e.g., "1 tree"). The stimuli are read in at line $\mathbf{1 3 0 .}$

\section{Screen Persistence}

One problem of microcomputer presentation is that CRT screens have various persistence times, making accurate timing of stimulus presentation difficult. This problem is easily circumvented by using the reverse video mode: the screen remains on except for those pixels that correspond to the stimulus. Thus, when the stimulus is removed, lights are turned back on, removing any chance of screen persistence. Using a backward mask also reduces the screen persistence problem, especially when displaying in normal video.

\section{Response Output File}

If a verbal response is to be scored for content, clearly no reaction time (RT) information is needed, and therefore no output file is required (delete lines 140 and 560-600). If the response is a keyboard keypress (e.g., same/different, yes/no, lexical decision, etc.), then the output file (FOUT\$) storing the data can contain several pieces of information: stimulus number, RT, the ASCII code of the key pressed, the designated VHF, and the stimulus word itself.

\section{Sync Pulse}

It is important with CRT presentation to time the stimulus onset to coincide with the return of the raster for a new screen scan, so that the RT can be linked consistently to the stimulus onset. The IBM color graphics card refreshes the screen 60 times per second. Of the $16.7 \mathrm{msec}$ required for each screen refresh, approximately $1 \mathrm{msec}$ is taken up by the raster returning to the top of the screen. It is during this period that the active screen page is switched (line 460) to the one with the stimulus and the clock timer is reset. The sync pulse, registered in bit 3 of PORT 986, is high when information is being sent to the screen. 


\section{Millisecond Clock Timer}

Tecmar provides a millisecond clock timer on many of its multifunction cards with a BASIC routine to read the clock times. With this routine, there may be some millisecond inaccuracy during the reading process because of rollover, where a millisecond value of 9 is read and then augmented, forcing a carry in the hundredths, before the rest of the time is read. The assembly language subroutine CLOCKRD.ASM (Listing 2) reads the clock in approximately $0.029 \mathrm{msec}$ and, if the time has changed during the reading process, the system reads it again. The result in this procedure is that the $\mathrm{RT}$ is rounded up when it is within $29 \mu \mathrm{sec}$ of the next millisecond.

If a real-time clock is unavailable, the user can substitute for one with a looping subroutine. For example, to time how long it takes for the subject to press a key, substitute Listing 3 for lines 650-670 in Listing 1. Note that line 655 requires a maximum reaction time (TIMEOUT) defined earlier in the program. The formula in line 670 converts the units to milliseconds, but the raw score LOOP can, of course, be used for higher resolution. Similarly, Listing 4 should substitute for lines $820-850$ to provide the wait subroutine. The formulas converting the loops to milliseconds are derived from compiling the code using the IBM PC BASIC Compiler, Version 1.00.

Please note an important limitation to not using a separate real-time clock. The timing of the subject's response cannot begin until the stimulus is off, assuming the experimenter wants the stimulus exposed for a brief period. In other words, the timing cannot start until all screen handling is done. Since VHF presentation must be for less than $200 \mathrm{msec}$ to be effective and since RT is normally greater than $400 \mathrm{msec}$, this poses no problem in the paradigm presented. However, this may not always be the case, and purchase of a real-time clock with millisecond reporting should be considered.

\section{Stimulus Duration}

The stimulus can be left on for times in multiples of $16.7 \mathrm{msec}$, referred to in the program as NSCANS, read in by the user (line 90). The intertrial interval is set in lines 525-526. The fixation-point duration is set in lines 320-340.

\section{Fixation Point}

The fixation point is currently set to appear with the stimulus. To disable it, remove line 380 . The postexposure field is currently blank. To maintain a fixation point continuously, remove the apostrophe in line 220 .

\section{Running the Program}

Since the stimuli and results are stored in RAM until the session is finished, a RAM disk is not needed. The DIMension statement (line 70) allows up to 256 stimuli. This can be increased as needed.

The program can be halted by pressing the space bar between trials and then continued by hitting any key. Pressing " $R$ " between trials restarts the program from the beginning. Pressing " $S$ " between trials stops the program and stores the data gathered thus far. Pressing " $Q$ " between trials stops the program without saving the data.

\section{HARDWARE REQUIREMENTS}

To run the program, one needs an IBM PC or compatible computer with IBM color graphics card, one disk drive, and $96 \mathrm{~K}$ of RAM.

\section{SOFTWARE REQUIREMENTS}

The following are needed: BASICA and BASIC compiler, MacroAssembler (optional), and Tecmar clock (optional).

\section{REFERENCE}

Diener, D., \& SMEE, W.P. (1984). Apple tachistoscope. Behavior Research Methods, Instruments, \& Computers, 16, 540-544.

\section{NOTE}

1. Tecmar, Inc., 6225 Cochran Rd., Cleveland, OH 44139. I have not been able to find another real-time clock for the IBM PC that permits millisecond access.

\section{LISTING 1}

The BASIC Main Program

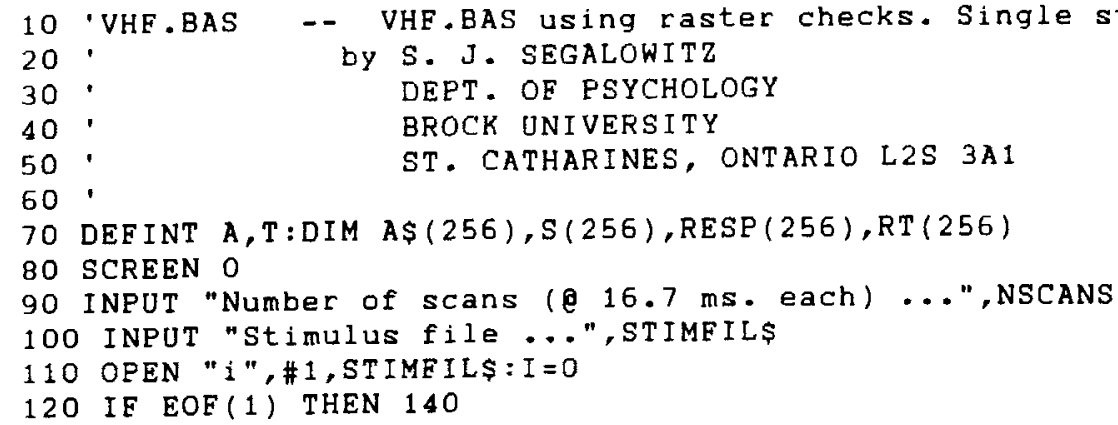


LISTING 1 (Continued)

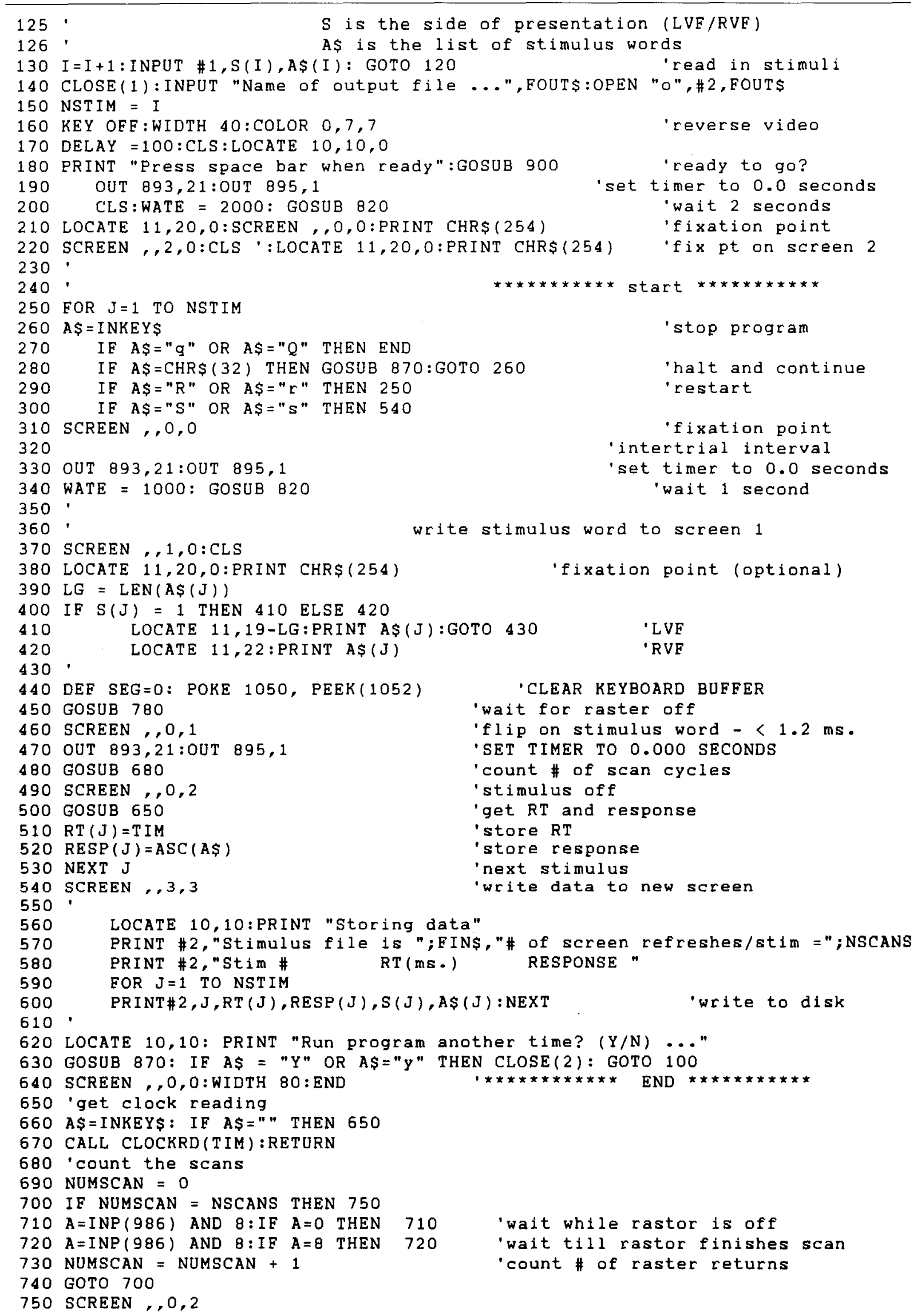




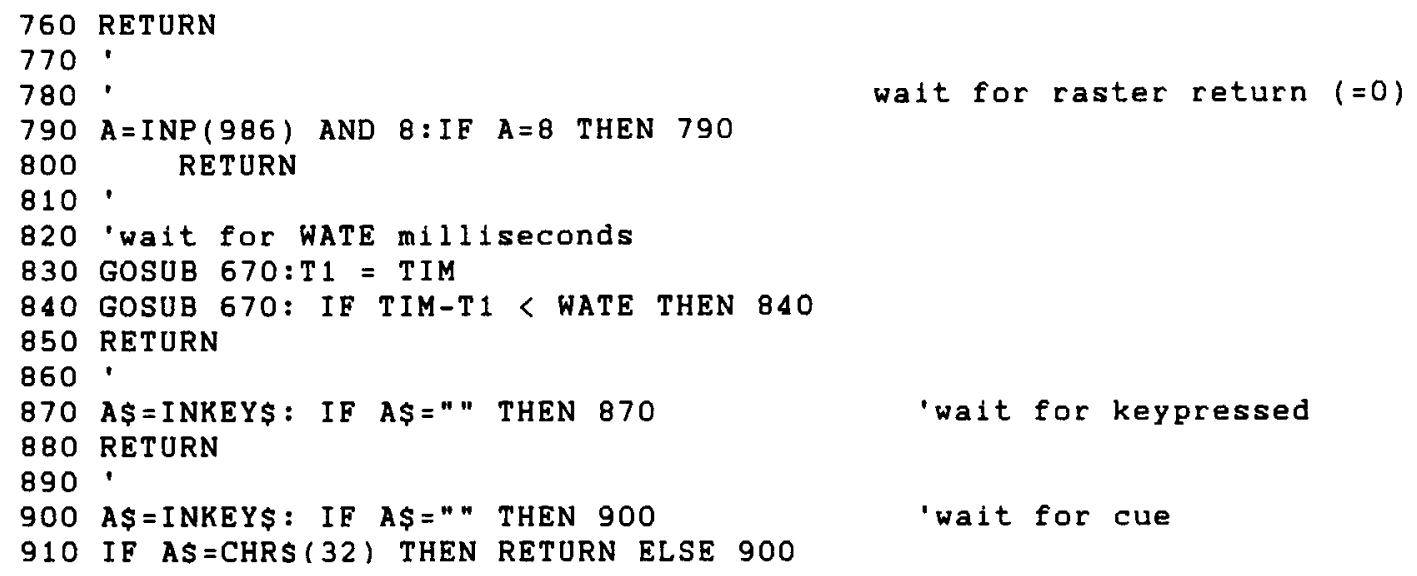

\section{LISTING 2}

Assembly Language Subroutine to Read the Time in Milliseconds from the Tecmar Clock

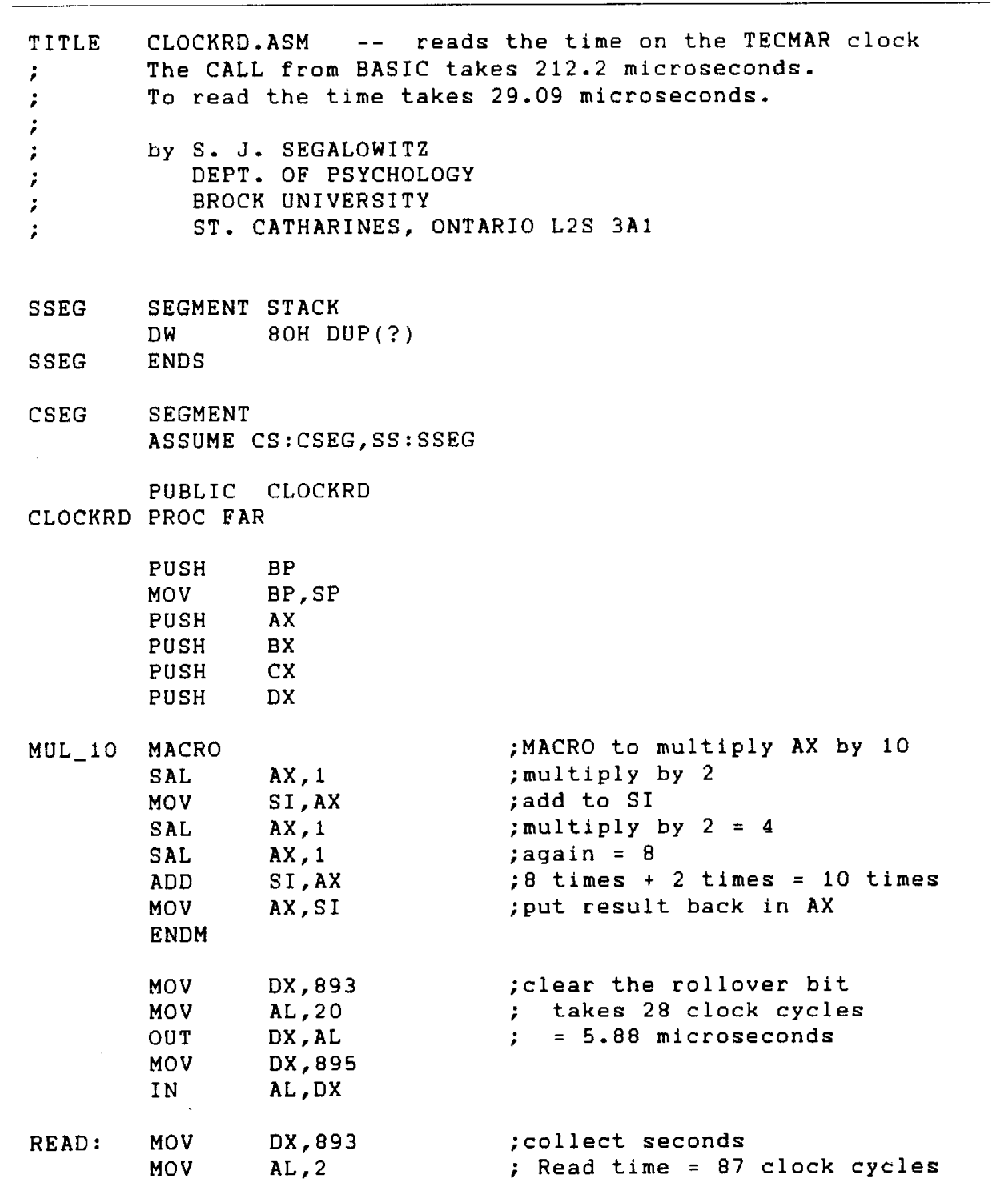


LISTING 2 (Continued)

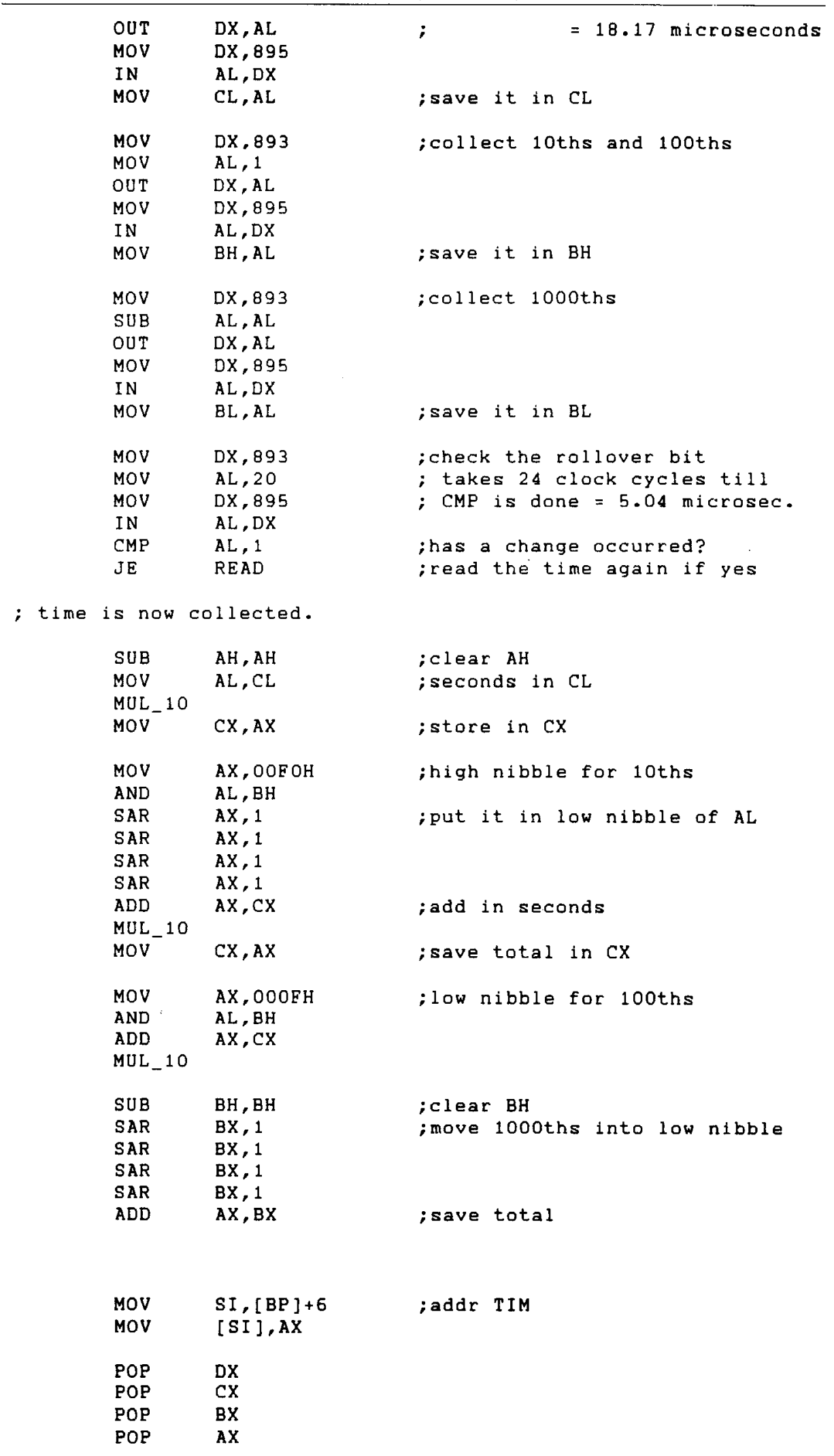




\begin{tabular}{lll} 
& POP \\
& RET 2 \\
CLOCKRD & ENDP \\
CSEG & ENDS \\
& END \\
\hline
\end{tabular}

\section{LISTING 3}

Reaction-Time Loop to be Used if No Real-Time Millisecond Clock is Available

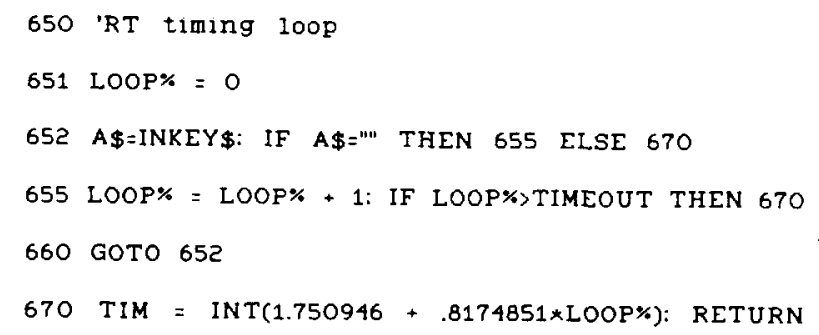

Note-Accuracy is within $1 \mathrm{msec}$ with times 100 to $2,000 \mathrm{msec}$.

\section{LISTING 4}

Timing Loop for Waiting a Specified Time if No Real-Time Clock is Available

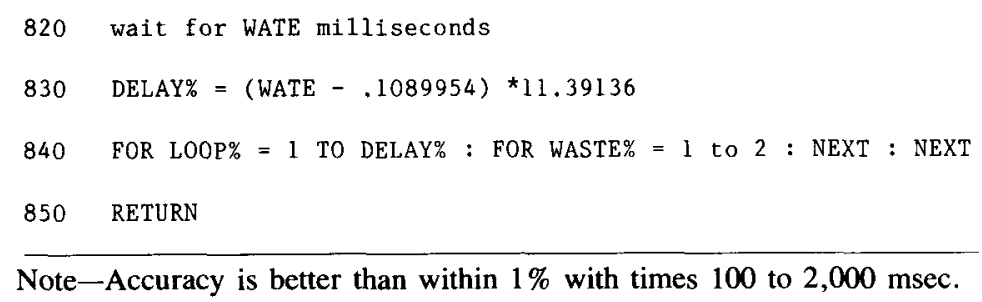

(Manuscript received September 12, 1986; revision accepted for publication January 30, 1987.) 